\title{
Ultrasound Microbubbles for Molecular Diagnosis, Therapy, and Theranostics
}

\author{
Fabian Kiessling ${ }^{1}$, Stanley Fokong ${ }^{1}$, Patrick Koczera ${ }^{1}$, Wiltrud Lederle ${ }^{1}$, and Twan Lammers ${ }^{1,2}$ \\ ${ }^{I}$ Department of Experimental Molecular Imaging, RWTH-Aachen University, Aachen, Germany; and ${ }^{2}$ Department of Targeted \\ Therapeutics, University of Twente, Enschede, The Netherlands
}

Ultrasound imaging is clinically established for routine screening examinations of breast, abdomen, neck, and other soft tissues, as well as for therapy monitoring. Microbubbles as vascular contrast agents improve the detection and characterization of cancerous lesions, inflammatory processes, and cardiovascular pathologies. Taking advantage of the excellent sensitivity and specificity of ultrasound for microbubble detection, molecular imaging can be realized by binding antibodies, peptides, and other targeting moieties to microbubble surfaces. Molecular microbubbles directed against various targets such as vascular endothelial growth factor receptor-2, vascular cell adhesion molecule 1, intercellular adhesion molecule 1, selectins, and integrins were developed and were shown in preclinical studies to be able to selectively bind to tumor blood vessels and atherosclerotic plaques. Currently, the first microbubble formulations targeted to angiogenic vessels in prostate cancers are being evaluated clinically. However, microbubbles can be used for more than diagnosis: disintegrating microbubbles emit acoustic forces that are strong enough to induce thrombolysis, and they can also be used for facilitating drug and gene delivery across biologic barriers. This review on the use of microbubbles for ultrasound-based molecular imaging, therapy, and theranostics addresses innovative concepts and identifies areas in which clinical translation is foreseeable in the near future.

Key Words: microbubbles; ultrasound; sonography; angiogenesis; drug delivery; theranostics

J Nucl Med 2012; 53:345-348

DOI: 10.2967/jnumed.111.099754

Ultrasound imaging is one of the most widespread diagnostic modalities used in clinics. Its application ranges from first-look examinations of the abdomen and other soft tissues to endosonography via the esophagus or the female genital tract and intravascular applications. Ultrasound examinations are usually performed without contrast agents, and the vascular information derived from color or power Doppler is generally sufficient to find the right diagnosis. However, gas-filled microbubbles as intravascular contrast agents have proven to be highly beneficial in some situations such as the characterization of liver lesions (1), the assessment of microcirculation in soft tissues and tumors (2), and the assessment of therapy response (3). Microbubbles are also routinely used to evaluate myocardial perfusion and heart function and to diagnose vesicoureteric reflux.

Over the last few years the sensitivity and specificity of ultrasound devices to detect microbubbles has improved steadily. In particular, the development of harmonic imaging has allowed for the application of microbubble-specific scan modes (e.g., amplitude modulation techniques or pulse inversion techniques). These are based on the fact that in comparison to soft tissues, insonated microbubbles have a much stronger nonlinear response, which can be used to suppress tissue background signals and to detect microbubbles. In comparison to destructive (e.g., Doppler-based) microbubble detection techniques, there is not much contribution of blood flow to the signal-particularly important when microbubbles are used to diagnose cardiovascular diseases.

Received Dec. 15, 2011; revision accepted Feb. 20, 2012.

For correspondence or reprints contact: Fabian Kiessling, Department of Experimental Molecular Imaging, University Aachen (RWTH), Pauwelsstrasse 20, 52074 Aachen, Germany.

E-mail: fkiessling@ukaachen.de

COPYRIGHT (c) 2012 by the Society of Nuclear Medicine, Inc.
High sensitivity and specificity of imaging modalities for the detection of contrast materials is an important precondition for molecular imaging. Thus, although restricted to the vascular compartment, molecular ultrasound imaging with microbubbles is promising, and much effort has been invested in generating targeted probes and determining detection techniques $(2,4,5)$. This review addresses the development of molecular ultrasound imaging on its way to the clinic (Fig. 1) and the use of microbubbles for thrombolysis, for facilitating drug and gene delivery across biologic barriers, and for theranostic applications (Fig. 2).

\section{MICROBUBBLES FOR MOLECULAR IMAGING}

Microbubble Overview

Microbubbles for ultrasound contrast enhancement usually have a diameter of between 1 and $4 \mu \mathrm{m}$, thus restricting them to the vascular compartment. Microbubbles have evolved from free gas bubbles in solution over bubbles stabilized by surfactants to bubbles with a shell made from phospholipids, proteins, or polymers. Microbubbles are generally synthesized by mechanical agitation, sonication, or the use of microfluidic devices. A detailed description of the different kinds of microbubbles and their synthesis procedures is beyond the scope of this review (2). Here, we focus on the production of microbubbles for molecular imaging. Tissue and cell specificity can be achieved by using passively or actively targeted microbubbles. Passive targeting refers to the use of the intrinsic properties of the microbubble shell to increase its affinity for a particular cell type or tissue. Albumin-shelled microbubbles, for example, were shown to bind to adherent leukocytes via cell-surface integrins or complement-mediated opsonization solely due to their shell composition $(2,4,5)$.

Active targeting refers to covalent or noncovalent attachment of specific targeting moieties to the microbubble shell, to allow for binding to specific receptors. This attachment can be achieved by a 1-step process, in which the targeting moieties are incorporated during microbubble synthesis. Such a 1-step process is suitable for molecules whose chemical properties are not affected by the harsh conditions encountered during microbubble synthesis. An important example is BR55, the first molecular ultrasound contrast agent to enter clinical trials, in which phospholipids preconjugated to a vascular endothelial growth factor receptor-2 (VEGFR2)binding peptide are mixed with peptide-free phospholipids to form microbubbles (6).

Alternatively, targeting ligands can also be attached to preformed microbubbles. This is most often achieved by use of the strong noncovalent interaction between strept(avidin) and biotin, which are generally bound to the microbubble surface and to a specific antibody or peptide using either carbodiimide chemistry (7) or stable thioether bonds (8).

Unwanted side effects for targeted microbubbles are expected to be comparable to those for nontargeted ones. These side effects include hypersensitivity and allergic reactions that occurred occasionally in patients. Additionally, myocardial side effects, headaches, and abdominal pain have been reported for some contrast agents, and temporary microembolisms have been discussed. Vancraeynest et al. (9) reported on a reversible myocardial injury induced by the destruction of targeted microbubbles in rats. Damage to blood vessels at the microvascular level is possible after microbubble destruction, when using high microbubble concentrations and long insonation times. Nevertheless, microbubbles are usually administered at low doses, and the incidence of side effects is generally much lower than that for CT and MRI contrast agents. 


\begin{tabular}{|c|c|c|}
\hline Diagnosis & & Therapy \\
\hline \multicolumn{3}{|c|}{ Microbubble (MB) / Nanobubble (NB), Nanodroplet (ND) } \\
\hline \multirow{2}{*}{ Targeted NB } & $\begin{array}{l}\text { Basic } \\
\text { research }\end{array}$ & \\
\hline & \multirow{3}{*}{$\begin{array}{l}\text { Preclinical } \\
\text { use }\end{array}$} & \\
\hline \multirow{2}{*}{$\begin{array}{l}\text { Various targeted MBs } \\
\text { Angiogenesis, (vascular) inflammation }\end{array}$} & & $\begin{array}{l}\text {-Drug/DNA/RNA loaded } \\
\text { MB, NB, ND }\end{array}$ \\
\hline & & $\begin{array}{l}\text { MBs supporting HIFU } \\
\text { MBs for vascular permeation } \\
\text { e.g. BBB opening, drug delivery }\end{array}$ \\
\hline $\begin{array}{l}\text { KDR-targeted soft shell MB } \\
\text { Prostate cancer delineation }\end{array}$ & $\begin{array}{l}\text { Clinical } \\
\text { evaluation }\end{array}$ & —Soft shell MBs for thrombolysis \\
\hline $\begin{array}{l}\text { Nontargeted MBs } \\
\text { (soft and hard shell) }\end{array}$ & $\begin{array}{l}\text { Routine } \\
\text { use }\end{array}$ & \\
\hline e.g. liver, prostate, head \& neck imagin & & \\
\hline
\end{tabular}

FIGURE 1. Diagram illustrating development stage of microbubbles, nanobubbles, and nanodroplets for diagnostic and therapeutic purposes. $\mathrm{HIFU}=$ high-intensity focused ultrasound; KDR = kinase domain receptor.

\section{Preclinical Applications and First Clinical Trial}

Molecular ultrasound with targeted microbubbles is predominantly used to monitor molecular markers expressed on the activated endothelium. In this context, the potential of targeted microbubbles for assessing tumor angiogenesis and inflammation has been intensively investigated predominantly in preclinical models, which are summarized in Table 1.

The most prominent molecular markers for the assessment of tumor angiogenesis and therapy effects by ultrasound are VEGFR2 (2) and $\alpha_{\mathrm{v}} \beta_{3}$ integrin (10). Both markers have been intensely addressed using various preclinical models. In angiogenic ovarian cancer xenografts, higher signal intensity was measured using dual-targeted microbubbles against VEGFR2 and $\alpha_{\mathrm{v}} \beta_{3}$ integrin than using single-targeted ones (10). Furthermore, microbubbles targeting the VEGF-VEGFR complex, VEGFR2, and endoglin were used to analyze angiogenic activity in pancreatic carcinoma xenografts and to monitor antiangiogenic or cytotoxic responses (11). Similarly, in squamous cell carcinoma xenografts treated with the matrix-metalloproteinase inhibitor prinomastat, significantly lower binding of VEGFR2- and $\alpha_{v} \beta_{3}$ integrin-binding microbubbles was observed (12). However, histologic analyses demonstrated that the lower signal intensities recorded in the treated tumors were due to a general decrease in vessel density and not to decreased marker expression on the tumor endothelium (12). These results strongly recommend combining functional and molecular ultrasound to elucidate whether an altered microbubble accumulation during therapy is due to a change in the relative blood volume or to alterations in marker expression.

Furthermore, molecular ultrasound was tested for monitoring activated endothelium during inflammation (e.g., in postischemic injury or atherosclerosis). Here, commonly addressed targets are E-selectin, P-selectin, vascular cell adhesion molecule 1 (VCAM-1), and intercellular adhesion molecule 1. E- and P-selectin-specific microbubbles showed a significantly enhanced retention in postischemic myocardium $(13,14)$, and the binding of VCAM1-specific microbubbles to plaque-containing inflamed endothelium correlated well with the stage of atherosclerosis (Figs. 1A-1D) (15).

In preclinical research, ligands are predominantly coupled to microbubbles via (strept)avidin-biotin, which might be problematic because of potential immunogenicity issues in humans. The first formulation for clinical application is BR55, that is, (strept)avidin-biotin-free soft shell microbubbles that target mouse and human VEGFR2. BR55 was found to strongly bind to the tumor endothelium of breast (16) and prostate cancer xenografts in rodents (17). BR55 was also successfully applied, with high sensitivity, for monitoring antiangiogenic therapy effects in human colon cancer xenografts (6). In addition, BR55 sensitively depicted the angiogenic status in 2 breast carcinoma xenografts with different degrees of aggressiveness (18). BR55 is currently being evaluated in a phase 0 clinical study for its ability to identify prostate cancers on the basis of their increased VEGFR2 expression (using a visual score in comparison with histopathologic analysis).

\section{MICROBUBBLES FOR THERAPY}

\section{Thrombolysis}

In the 1970s, Truebestein et al. (19) for the first time demonstrated that high-frequency ultrasound can be used to dissolve thrombi. In 2004, Alexandrov et al. (20) confirmed the potential of ultrasound-mediated thrombolysis, showing in a cohort of over 100 stroke patients pretreated with tissue plasminogen activator (tPA) that the subsequent administration of transcranial Doppler ultrasound improves arterial recanalization. A decade before this landmark achievement, Tachibana et al. (21) had already demonstrated ex vivo that the coadministration of microbubbles can further enhance the thrombolytic activity of ultrasound in combination with pharmaceutical thrombolysis. This effect is largely the result of stable and/or inertial microbubble oscillation and cavitation, leading to clot destruction. Extending this strategy to patients, Molina et al. (22) showed in 2006 that the addition of

FIGURE 2. Microbubbles for molecular imaging, therapy, and theranostics. (A-D) Representative images of the aortic arch of an ApoE-knockout mouse on high-cholesterol diet obtained using 2-dimensional B-mode imaging (A), pulsed-wave Doppler ultrasound (B), and molecular ultrasound using VCAM-1-targeted (C) and control (D) microbubbles (15). (E-H) Binding $(E-G)$ and quantification $(H)$ of control $(E)$, RGD-targeted (F), and VEGFR2-targeted (G) microbubbles to angiogenic tumor endothelium (12). (I) Spectral waveform visualization of arterial recanalization in patient with middle cerebral artery occlusion before (left) and after (right) microbubble-enhanced sonothrombolysis (22). (J) Quantification of complete, partial, and no MCA recanalization on treatment with $t P A$, tPA plus ultrasound, and tPA plus ultrasound plus microbubbles (22). (K-M) Highly efficient
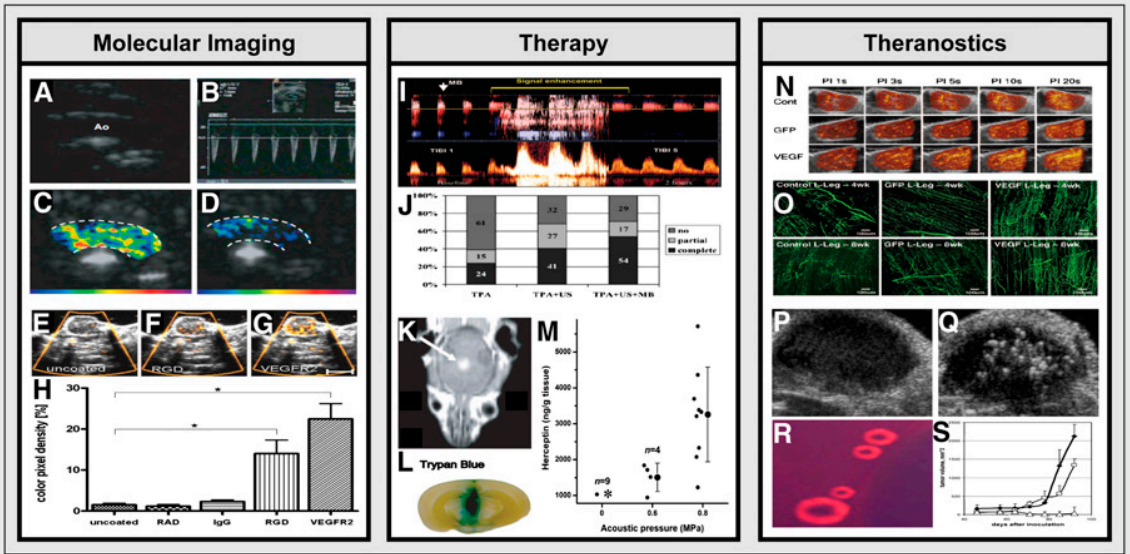

delivery of the MRI contrast agent gadopentetate dimeglumine (K), trypan blue $(\mathrm{L})$ and trastuzumab (M) of the BBB upon microbubble-enhanced focused ultrasound treatment at 2 different acoustic pressures (middle column: $0.6 \mathrm{MPa}$; right column: $0.8 \mathrm{MPa}$ ) (reprinted with permission from Proc Natl Acad Sci USA. 2006;103:11719-11723, Copyright (2012) National Academy of Sciences, USA (23)). (N-O) Combination of microbubbles with ultrasound (at various different pulsing intervals) improves the delivery of plasmid DNA encoding for VEGF to ischemic hind limb muscle in rats, leading to improved perfusion $(\mathrm{N})$ and to dense proliferation of neovessels, with abundant bridging arterioles $(\mathrm{O})(26)$. (P-S) Doxorubicin-containing nanobubbles coalesce into microbubbles at physiologic temperatures $(R)$, they can be visualized upon extravasation into subcutaneous tumor xenografts using B-mode imaging (P: preinjection; $\mathrm{Q}: 4 \mathrm{~h}$ after intravenous injection), and they can be used in combination with focused ultrasound to induce highly efficient tumor growth inhibition (S) (28). Images are adapted from indicated references with permission of publishers. 
TABLE 1

Common Ultrasound Molecular Imaging Targets, Conjugation Methods, and Applications

\begin{tabular}{|c|c|c|c|}
\hline Target & Ligand (conjugation method) & Application & Reference \\
\hline \multirow[t]{3}{*}{ VEGF receptor type 2} & Anti-VEGFR-2 mAb* & Preclinical & $(10-12)$ \\
\hline & Single-chain VEGF ${ }^{\dagger}$ & Preclinical & (8) \\
\hline & Heterodimeric lipopeptide ${ }^{\dagger}$ & Preclinical/clinical (prostate cancer) & $(4,16-18)$ \\
\hline \multirow[t]{7}{*}{$a_{v} \beta_{3}$-integrin } & CRGD peptide ${ }^{\star}$ & Preclinical & (12) \\
\hline & cRRL peptide ${ }^{\star}$ & Preclinical & (29) \\
\hline & Anti-integrin $a_{v}$-chain $m A b^{*}$ & Preclinical & (30) \\
\hline & Anti-RGD-containing disintegrin echistatin $\mathrm{mAb} \mathrm{b}^{\star}$ & Preclinical & (30) \\
\hline & Echistatin $^{*}$ & Preclinical & (31) \\
\hline & Knottin peptide binding $a_{v} \beta_{3}$-integrin ${ }^{*}$ & Preclinical & (32) \\
\hline & Cyclic RGD peptide ${ }^{\dagger}$ & Preclinical & (33) \\
\hline ICAM-1/NCAM-1 & Anti-ICAM-1 and -VCAM-1 $m A b^{*}$ & Preclinical & $(15,34)$ \\
\hline CD105 & Anti-CD105 $\mathrm{mAb}^{*}$ & Preclinical & $(11,35)$ \\
\hline$P$ or $E$ selectin & Polymeric sulfo-Lewis- $\mathrm{x}^{*}$ & Preclinical & $(13,14)$ \\
\hline
\end{tabular}

microbubbles to ultrasound and to initial tPA-based pharmaceutical thrombolysis also works well clinically. In patients with middle cerebral artery (MCA) occlusion, they demonstrated that the triple combination of tPA, ultrasound, and microbubbles was significantly more effective than tPA plus ultrasound and than tPA alone (Figs. 2I-2J). Besides the findings for stroke, promising findings for microbubble-enhanced ultrasound-mediated fibrinolysis have also been reported for declotting thrombosed arteriovenous dialysis grafts in dogs, as well as for improving periinfarction microvascular flow in acute ST-segment elevation myocardial infarction in pigs. Together, these findings convincingly demonstrate that microbubbles hold significant potential for improving the efficacy of clinically relevant combinations of pharmaceutical and ultrasound-based thrombolysis.

\section{Drug Delivery}

Besides showing potential for thrombolysis, the application ultrasound plus microbubbles also holds significant potential for improving drug delivery across biologic barriers. Pioneering proof of this principle has been provided by Kinoshita et al. (23), who showed that microbubbles combined with focused ultrasound facilitate the delivery of trastuzumab across the blood-brain barrier (BBB). In this context, focused ultrasound refers to the concentration of acoustic energy on a focal spot a few millimeters in diameter. Depending on the energy used, biologic effects such as heat-induced coagulation can result. However, acoustic pressures induced by microbubble destruction with relatively low ultrasound energies can also temporarily increase blood vessel and/or cell membrane permeability in the insonated region, thereby enhancing local drug delivery. In mice, Kinoshita et al. initially demonstrated that the application of microbubbles together with focused ultrasound enhanced the delivery of the MRI contrast agent gadopentetate dimeglumine across the BBB (Fig. 2K), as well as that of trypan blue (Fig. 2L). Subsequently, they quantified the concentration of trastuzumab delivered across the BBB and observed that although the concentration was below the detection threshold in 8 of 9 cases without the simultaneous application of microbubbles and ultrasound, the concentration steadily increased when microbubbles plus ultrasound were used at pressures of $0.6 \mathrm{MPa}$ and $0.8 \mathrm{MPa}$ (Fig. 2M).

These findings confirm and extend previous findings on the ability of microbubbles to enhance the ultrasound-mediated delivery of therapeutic moieties across biologic barriers, including the BBB, extracranial endothelial layers, and cellular membranes (5). This mechanism, generally referred to as sonoporation, is expected to rely on several different physicochemical and physiological mechanisms, including, for example, on the generation of microjets (which might lead to transient pores in endothelial and cellular membranes), on the production of intracellular reactive oxygen species (which might also contribute to membrane permeability), and on the generation of heat (which might affect phospholipid and membrane fluidity) (24). Besides showing potential for facilitating the delivery of standard drugs to target cells, sonoporation has also been shown to hold significant potential for improving the delivery and the efficacy of genetic interventions based, for example, on plasmid DNA and on small interfering RNA $(5,24)$.

\section{MICROBUBBLES FOR THERANOSTICS}

Theranostics refers to the combination of disease diagnosis and therapy. Theranostic strategies range from setups in which patients are preselected on the basis of initial target site accumulation studies using a given diagnostic radionuclide-labeled antibody, followed by subsequent radioimmunotherapy with the same antibody coupled to a therapeutic radionuclide, to setups in which imaging agents and (chemo-) therapeutics are combined within a single micro- or nanomedicine formulation (25). In principle, microbubbles hold significant potential for theranostic applications, given their propensity to be visualized in vivo with extremely high sensitivity, their ability to improve drug delivery across biologic barriers, and the possibility of loading therapeutic molecules into or onto their shell. Thus far, however, only very few studies have evaluated such possibilities.

A pioneering proof-of-principle study in this regard has been published by Leong-Poi et al. (26), who used cationic microbubbles to complex plasmid DNA encoding for VEGF to induce therapeutic arteriogenesis in chronically ischemic skeletal muscle and who showed that these theranostic microbubbles were able to enhance the expression of VEGF in ischemic hind limbs in rats, to increase microvessel density, and to thereby improve microvascular blood flow (Figs. 2N-2O). It should be mentioned, though, that the cationic VEGF-plasmid DNA-containing microbubbles (MP1950; lipid-shelled microbubbles between 3.9 and $5.4 \mu \mathrm{m}$ ) were not used to visualize blood flow but merely to deliver VEGF into target cells. It can be reasoned, however, that when one is intending to administer multiple doses of MP1950, microbubbles might well be suited to directly visualize the therapeutic effects of the previous injection.

Similar semitheranostic efforts have recently been reported by Phillips et al. (27), who used rapamycin-loaded microbubbles plus ultrasound to locally reduce neointima formation after carotid balloon injury but who did not use the same formulation to monitor the degree of stenosis or treatment-induced improvements in perfusion. Truly theranostic efforts have been undertaken by Rapoport et al. (28), who loaded doxorubicin into perfluoropentane-based nanobubbles, which on intravenous injection first extravasated into the tumor interstitium, then coalesced into microbubbles at physiologic temperatures, then were visualized at the target site using ultrasound, and then were destroyed to release the drug, eventually inducing highly effective tumor growth inhibition (Figs. 2P-2S).

Several analogous theranostic approaches are currently being undertaken both in our laboratory and in other laboratories to extend these efforts, using, for example, chemotherapy-loaded microbubbles targeted to tumor blood vessels, visualizing and quantifying their target site localization, and destroying them locally to induce drug release, drug delivery across biologic barriers, and drug efficacy only at the target site. In addition, when such agents are administered more than once, as will likely be the case clinically, follow-up injections using the same theranostic microbubble formulations would enable not only highly efficient targeted and triggered drug delivery but also simultaneous feedback on the therapeutic efficacy of the previous administration, via both functional perfusion monitoring and molecular ultrasound imaging. 


\section{CONCLUSION}

Preclinically, several different antibody- or peptide-targeted microbubbles have been successfully tested for visualizing receptors overexpressed on tumor blood vessels and on atherosclerotic plaques. Clinically, the first molecularly targeted microbubble formulation has recently entered phase 0 trials, for the diagnosis and localization of prostate cancer. In addition, microbubbles have been used for thrombolysis, for enhancing drug delivery across biologic barriers, and for combining disease diagnosis and therapy. In more than 100 patients with stroke, for instance, microbubbles have been successfully combined with transcranial Doppler ultrasound and with tPA to induce middle cerebral artery recanalization, which underlines their therapeutic potential. Moreover, taking the diagnostic and therapeutic potential of microbubbles (including their ability to carry and facilitate site-specific drug delivery) into account, one can conclude that ultrasound imaging in combination with microbubbles provides a versatile tool for molecular imaging, therapy, and theranostics.

\section{ACKNOWLEDGMENT}

We acknowledge financial support by the DFG (KI 1072/5-1, KI 1072/ 8-1 and LA 2937-1/1), by the EC (COST-Action TD1004) and by Hightech.NRW (ForSaTum), and apologize to colleagues whose work could not be cited due to space constraints. No other potential conflict of interest relevant to this article was reported.

\section{REFERENCES}

1. Cosgrove D, Lassau N. Imaging of perfusion using ultrasound. Eur J Nucl Med Mol Imaging. 2010;37(suppl 1):S65-S85.

2. Kiessling F, Huppert J, Palmowski M. Functional and molecular ultrasound imaging: concepts and contrast agents. Curr Med Chem. 2009;16:627-642.

3. Lassau N, Chami L, Benatsou B, Peronneau P, Roche A. Dynamic contrastenhanced ultrasonography (DCE-US) with quantification of tumor perfusion: a new diagnostic tool to evaluate the early effects of antiangiogenic treatment. Eur Radiol. 2007;17(suppl 6):F89-F98.

4. Deshpande N, Needles A, Willmann JK. Molecular ultrasound imaging: current status and future directions. Clin Radiol. 2010;65:567-581.

5. Ferrara K, Pollard R, Borden M. Ultrasound microbubble contrast agents: fundamentals and application to gene and drug delivery. Annu Rev Biomed Eng. 2007;9:415-447.

6. Pysz MA, Foygel K, Rosenberg J, Gambhir SS, Schneider M, Willmann JK. Antiangiogenic cancer therapy: monitoring with molecular US and a clinically translatable contrast agent (BR55). Radiology. 2010;256:519-527.

7. Palmowski M, Morgenstern B, Hauff $\mathrm{P}$, et al. Pharmacodynamics of streptavidincoated cyanoacrylate microbubbles designed for molecular ultrasound imaging. Invest Radiol. 2008;43:162-169.

8. Anderson CR, Rychak JJ, Backer M, Backer J, Ley K, Klibanov AL. scVEGF microbubble ultrasound contrast agents: a novel probe for ultrasound molecular imaging of tumor angiogenesis. Invest Radiol. 2010;45:579-585.

9. Vancraeynest D, Havaux X, Pasquet A, et al. Myocardial injury induced by ultrasound-targeted microbubble destruction: evidence for the contribution of myocardial ischemia. Ultrasound Med Biol. 2009;35:672-679.

10. Willmann JK, Lutz AM, Paulmurugan R, et al. Dual-targeted contrast agent for US assessment of tumor angiogenesis in vivo. Radiology. 2008;248:936-944.

11. Korpanty G, Carbon JG, Grayburn PA, Fleming JB, Brekken RA. Monitoring response to anticancer therapy by targeting microbubbles to tumor vasculature. Clin Cancer Res. 2007;13:323-330.

12. Palmowski M, Huppert J, Ladewig G, et al. Molecular profiling of angiogenesis with targeted ultrasound imaging: early assessment of antiangiogenic therapy effects. Mol Cancer Ther. 2008;7:101-109.
13. Kaufmann BA, Lewis C, Xie A, Mirza-Mohd A, Lindner JR. Detection of recent myocardial ischaemia by molecular imaging of P-selectin with targeted contrast echocardiography. Eur Heart J. 2007;28:2011-2017.

14. Villanueva FS, Lu E, Bowry S, et al. Myocardial ischemic memory imaging with molecular echocardiography. Circulation. 2007;115:345-352.

15. Kaufmann BA, Sanders JM, Davis C, et al. Molecular imaging of inflammation in atherosclerosis with targeted ultrasound detection of vascular cell adhesion molecule-1. Circulation. 2007;116:276-284.

16. Pochon S, Tardy I, Bussat P, et al. BR55: a lipopeptide-based VEGFR2-targeted ultrasound contrast agent for molecular imaging of angiogenesis. Invest Radiol. 2010;45:89-95.

17. Tardy I, Pochon S, Theraulaz M, et al. Ultrasound molecular imaging of VEGFR2 in a rat prostate tumor model using BR55. Invest Radiol. 2010;45:573-578.

18. Bzyl J, Lederle W, Rix A, et al. Molecular and functional ultrasound imaging in differently aggressive breast cancer xenografts using two novel ultrasound contrast agents (BR55 and BR38). Eur Radiol. 2011;21:1988-1995.

19. Trübestein G, Engel C, Etzel F, Sobbe A, Cremer H, Stumpff U. Thrombolysis by ultrasound. Clin Sci Mol Med Suppl. 1976;3:697s-698s.

20. Alexandrov AV, Molina CA, Grotta JC, et al. Ultrasound-enhanced systemic thrombolysis for acute ischemic stroke. N Engl J Med. 2004;351:2170-2178.

21. Tachibana K, Tachibana S. Albumin microbubble echo-contrast material as an enhancer for ultrasound accelerated thrombolysis. Circulation. 1995;92:1148-1150.

22. Molina CA, Ribo M, Rubiera M, et al. Microbubble administration accelerates clot lysis during continuous 2-MHz ultrasound monitoring in stroke patients treated with intravenous tissue plasminogen activator. Stroke. 2006;37:425-429.

23. Kinoshita M, McDannold N, Jolesz FA, Hynynen K. Noninvasive localized delivery of Herceptin to the mouse brain by MRI-guided focused ultrasound-induced bloodbrain barrier disruption. Proc Natl Acad Sci USA. 2006;103:11719-11723.

24. Hernot S, Klibanov AL. Microbubbles in ultrasound-triggered drug and gene delivery. Adv Drug Deliv Rev. 2008;60:1153-1166.

25. Lammers T, Aime S, Hennink WE, Storm G, Kiessling F. Theranostic nanomedicine. Acc Chem Res. 2011;44:1029-1038.

26. Leong-Poi H, Kuliszewski MA, Lekas M, et al. Therapeutic arteriogenesis by ultrasound-mediated VEGF165 plasmid gene delivery to chronically ischemic skeletal muscle. Circ Res. 2007;101:295-303.

27. Phillips LC, Dhanaliwala AH, Klibanov AL, Hossack JA, Wamhoff BR. Focused ultrasound-mediated drug delivery from microbubbles reduces drug dose necessary for therapeutic effect on neointima formation: brief report. Arterioscler Thromb Vasc Biol. 2011;31:2853-2855.

28. Rapoport N, Gao Z, Kennedy A. Multifunctional nanoparticles for combining ultrasonic tumor imaging and targeted chemotherapy. J Natl Cancer Inst. 2007; 99:1095-1106.

29. Weller GE, Wong MK, Modzelewski RA, et al. Ultrasonic imaging of tumor angiogenesis using contrast microbubbles targeted via the tumor-binding peptide arginine-arginine-leucine. Cancer Res. 2005;65:533-539.

30. Leong-Poi H, Christiansen J, Klibanov AL, Kaul S, Lindner JR. Noninvasive assessment of angiogenesis by ultrasound and microbubbles targeted to alpha(v)integrins. Circulation. 2003;107:455-460.

31. Ellegala DB, Leong-Poi H, Carpenter JE, et al. Imaging tumor angiogenesis with contrast ultrasound and microbubbles targeted to alpha(v)beta3. Circulation. 2003; $108: 336-341$

32. Willmann JK, Kimura RH, Deshpande N, Lutz AM, Cochran JR, Gambhir SS. Targeted contrast-enhanced ultrasound imaging of tumor angiogenesis with contrast microbubbles conjugated to integrin-binding knottin peptides. J Nucl Med. 2010;51:433-440.

33. Anderson $\mathrm{CR}, \mathrm{Hu} \mathrm{X}$, Zhang $\mathrm{H}$, et al. Ultrasound molecular imaging of tumor angiogenesis with an integrin targeted microbubble contrast agent. Invest Radiol. 2011;46:215-224.

34. Villanueva FS, Jankowski RJ, Klibanov S, et al. Microbubbles targeted to intercellular adhesion molecule-1 bind to activated coronary artery endothelial cells. Circulation. 1998;98:1-5.

35. Fonsatti E, Altomonte M, Nicotra MR, Natali PG, Maio M. Endoglin (CD105): a powerful therapeutic target on tumor-associated angiogenetic blood vessels. Oncogene. 2003;22:6557-6563. 